\title{
CONTROL QUÍMICO DE MALEZAS EN FRIJOL (Phaseolus vulgaris L.) EN EL ESTADO DE VERACRUZ ${ }^{1}$
}

\author{
Valentín A. Esquivel ${ }^{2}$, Octavio Cano ${ }^{2}$, Ernesto López ${ }^{2}$
}

\begin{abstract}
RESUMEN
Control químico de malezas en frijol (Phaseolus vulgaris L.) en el estado de Veracruz. En los ciclos agrícolas 199091 y 1991-92, se condujeron dos experimentos de evaluación de herbicidas con el objeto de determinar los mejores tratamientos preemergentes y/o postemergentes en el control de las especies de malezas dominantes en el cultivo de frijol de humedad residual. Los experimentos se establecieron en la región central del estado de Veracruz y tuvieron un diseño experimental de bloques al azar, con 12 tratamientos y cuatro repeticiones. La flor amarilla (Melampodium divari-catum) y el coquillo (Cyperus rotundus) se presentaron como especies dominantes en las dos localidades, mientras que la leche de sapo (Euphorbia heterophylla) y el zacate Johnson (Sorghum halepense) fueron dominantes en una localidad cada uno. En preemergencia, los mejores controles de $M$. divaricatum se obtuvieron con alaclor (2 kg i.a./ha) y linurón ( $2 \mathrm{~kg} / \mathrm{ha}$ ), siendo también este último tratamiento eficiente para controlar $E$. heterophylla. En postemergencia, fomesafén $(0,25 \mathrm{~kg} / \mathrm{ha})$ tuvo un eficiente control de estas dos especies. Ninguno de los tratamientos controló a $C$. rotundus, y $S$. halepense sólo fue controlado con el único tratamiento que incluyó al herbicida fluazifop-butil. Linurón y alaclor ocasionaron toxicidad al frijol cuando la humedad residual del terreno fue alta, sin embargo, al contrario de estos dos herbicidas, fomesafén causó mayor toxicidad cuando la humedad residual fue baja.
\end{abstract}

\begin{abstract}
Chemical weed control in dry beans (Phaseolus vulgaris $L_{\text {.) }}$ in the state of Veracruz México. Two experiments wiht herbicides were carried out during the Fall-Winter seasons of 1990-91 and 1991-92. The objective was to determine the best preemergence and/or postemergence herbicide treatments for controlling the dominant weed species in the residual moisture growing sistem of beans. The experiments were established in the central area of the state of Veracruz, Mexico.In both experiments, treatments were distributed in a CRBD with 12 treatments and four replications. Melampodium divaricatum and Cyperus rotundus were the dominant weed species in both experimental sites. Euphorbia heterophylla and Sorghum halepense were dominant in one location each. In preemergence, the maximum control of $M$. divaricatum was obtained with alachlor ( $2 \mathrm{~kg}$ a.i./ha) and linuron ( $2 \mathrm{~kg} / \mathrm{ha})$, the last herbicide was also efficient to control E. heterophylla. In postemergence, fomesafen $(0.25 \mathrm{~kg} / \mathrm{ha})$ showed an efficient control of the two dominant weed species. No treatment controlled $C$. rotundus, and S. halepense was only controlled with a treatment included fluazifop-butyl. Linuron and alachlor caused toxicity to the bean plants when the residual moisture of the soil was high. However, and contrary to the response observed with these two herbicides, fomesafen caused a higher toxicity when the residual moisture in the soil was low.
\end{abstract}

\section{INTRODUCCIÓN}

En las siembras de frijol que se establecen en el estado de Veracruz, México, por lo general, el control de malezas se lleva a cabo manualmente, mediante el empleo de azadones o "tarpalas", que es un tipo de azadón recto usado por los agricultores del centro del estado (Cano y López, 1996). Las malezas son eliminadas solamente en una ocasión durante el ciclo del cultivo, entre los 25 y 40 días después de la emergencia.
Información proveniente de experimentos de competencia entre el frijol y las malezas, indica que este cultivo requiere de un período inicial de limpieza de al menos 30 días, y que en algunos casos, los daños por competencia pueden comenzar desde los primeros 20 días (Agundis, Valtierra y Castillo 1962; Barreto, 1970). Lo anterior indica que el bajo rendimiento de frijol que se obtiene en el estado (500-600 kg/ha), es en buena parte determinado por la competencia que sufre este cultivo debido a la eliminación tardía e ineficiente de la maleza.

Presentado en la XLI Reunión Anual del PCCMCA en El Salvador, Centroamérica. 27 al 31 de marzo, 1996.

2 Investigador de Malezas y su Control, Investigadores de Leguminosas comestibles. Campo Experimental Cotaxtla. CIRGOT. INIFAP. SAGAR. Apdo.Postal 429, 91700, Veracruz, Ver. México. 
Se ha determinado que para evitar los efectos de la competencia, el frijol debe deshierbarse en dos ocasiones; la primera limpia debe realizarse entre los 10 y 15 días después de la emergencia (DDE) y la segunda entre los 20 y 30 DDE (Esqueda y Durán, 1992).

Una alternativa al control manual o mecánico de la maleza, es la aplicación de productos químicos. En frijol existen varios herbicidas selectivos que pueden ser utilizados en preemergencia o postemergencia. Entre los herbicidas preemergentes, destacan por su eficiencia en el control de malezas y selectividad al cultivo, linurón (Obando, 1981), alaclor (Wilson, Wicks y Fenster, 1980) y la mezcla de alaclor + cianazina (Esqueda , Acosta y Dúran, 1981).

En postemergencia, herbicidas como bentazón (García y Barrales, 1988) y fomesafén (Romero y Alemán, 1990) representan una buena alternativa para el control de la maleza de hoja ancha. Fluazifop-butil es utilizado cuando el problema es la infestación de gramíneas tanto anuales como perennes (Thomson, 1993), siendo comúnmente aplicado en mezcla con fomesafén para combatir poblaciones de malezas tanto de hoja ancha como gramíneas (Romero y Alemán, 1990).
Debido a que el uso de herbicidas puede ser una buena opción para el control de las malezas del frijol en el estado de Veracruz, se establecieron dos experimentos de evaluación de herbicidas, con el objetivo de determinar él o los tratamientos selectivos más eficientes para controlar las malezas en frijol de humedad residual.

\section{MATERIALES Y METODOS}

Se establecieron dos experimentos en condiciones de humedad residual en el ejido Ignacio de la Llave, Mpio. de Ignacio de la Llave, Ver., en los ciclos 1990-91 y 1991-92, respectivamente.

En ambos casos, el diseño experimental fue de bloques al azar con 12 tratamientos (Cuadro 1) y cuatro repeticiones. Las parcelas experimentales estuvieron constituidas por cinco surcos de $6 \mathrm{~m}$ de longitud y una separación de 0,6 m entre ellos. Como parcela útil se tomaron los tres surcos centrales a los que se les eliminó $0,5 \mathrm{~m}$ en ambos extremos.

El frijol se sembró a una densidad equivalente a 250,000 plantas/ha, utilizándose las variedades Jamapa y

Cuadro 1. Tratamientos de los experimentos de evaluación de herbicidas en frijol de humedad residual en el estado de Veracruz. Cirgoc. Inifap. Sagar.

\begin{tabular}{lllll}
\hline Tratamiento & \multicolumn{1}{c}{$\begin{array}{c}\text { Dosis } \\
\text { (kg i.a./ha) }\end{array}$} & $\begin{array}{c}\text { Epoca de } \\
\text { aplicación }\end{array}$ & \multicolumn{2}{c}{ Ciclo de aplicación } \\
\cline { 4 - 5 } & 0,44 & PRE & 1990-91 & X91-92 \\
\hline Alaclor & 1,5 & PRE & $\mathrm{X}$ & \\
Alaclor & 2 & PRE & $\mathrm{X}$ & $\mathrm{X}$ \\
Alaclor & 2,5 & PRE & $\mathrm{X}$ & \\
Alaclor & 1 & PRE & $\mathrm{X}$ & $\mathrm{X}$ \\
Linurón & 1,5 & PRE & $\mathrm{X}$ & $\mathrm{X}$ \\
Linurón & 2 & PRE & $\mathrm{X}$ & $\mathrm{X}$ \\
Linurón & 6 & PRE & $\mathrm{X}$ & \\
Clortal-dimetil & 7,5 & PRE & & \\
Clortal-dimetil & $1+0.5$ & PRE & $\mathrm{X}$ & $\mathrm{X}$ \\
Alaclor + linurón & $1.5+0.5$ & PRE & $\mathrm{X}$ & $\mathrm{X}$ \\
Alaclor + linurón & 0,187 & 15 DDE & $\mathrm{X}$ & \\
Fomesafén & 0,25 & 15 DDE & $\mathrm{X}$ & $\mathrm{X}$ \\
Fomesafén & 0,375 & 15 DDE & $\mathrm{X}$ & \\
Fomesafén & 0,25 & 15 DDE & & \\
Fluazifop-butil + fomesafén & $\mathrm{X}$ & $\mathrm{X}$ & $\mathrm{X}$ & \\
Testigo limpio & $\mathrm{X}$ & $\mathrm{X}$ & & \\
Testigo enhierbado & & &
\end{tabular}

*/ $\mathrm{PRE}=$ preemergencia $; \mathrm{DDE}=$ días después de la emergencia 
Negro Cotaxtla 91 en los experimentos del ciclo O-I 1990-91 y O-I 1991-92, respectivamente.

Los herbicidas se aplicaron con una bomba motorizada de mochila, equipada con boquillas Tee-jet 8003 , las cuales proporcionaron un gasto de agua de 284 1/ha. Solamente se aplicaron los tres surcos centrales de cada parcela experimental, dejando el primero y último surco como testigos laterales enhierbados.

La densidad de población de malezas se cuantificó utilizando cuadrantes de 0,5 x 0,5 m, lanzados al azar sobre las parcelas correspondientes a los testigos enhierbados. Los conteos se llevaron a cabo a los 15 y 30 días después de la emergencia (DDE).

La evaluación de control de cada especie de maleza dominante se efectuó en forma visual, utilizando la escala de 0-100\%, en donde cero significó que el herbicida no tuvo ningún efecto en la especie evaluada, y 100, que esa especie fue completamente eliminada. En el experimento del ciclo O-I 1990-91, las evaluaciones se realizaron a los 20 y 30 días después de la aplicación (DDA) de los herbicidas, mientras que en el experimento del ciclo 1991-92, las evaluaciones se efectuaron a los 25 y 45 DDA para los herbicidas preemergentes y a los 15 y 30 DA para los herbicidas postemergentes.

Los efectos tóxicos en el frijol fueron evaluados visualmente en las mismas fechas en que el control de malezas fue evaluado. Los daños también fueron cuantificados mediante el uso de la escala 0-100\%.

Al tiempo de la cosecha se determinó el rendimiento de frijol al $14 \%$ de humedad. Se realizaron análisis de varianza del rendimiento de grano, y como prueba de significancia se utilizó Duncan $(0,05)$.

\section{RESULTADOS Y DISCUSIÓN}

En cada uno de los sitios experimentales se presentaron nueve especies de malezas, siendo su densidad de población mayor en el experimento establecido en el ciclo 1990-91 que en el ciclo 1991-92. El total de especies de malezas identificadas en los dos experimentos fue de 14 , pertenecientes a siete familias botánicas.

La flor amarilla (M. divaricatum) y el coquillo ( $C$. rotundus) se presentaron como especies dominantes en ambos experimentos. En el ciclo 1990-91, también la leche de sapo (E. heterophylla) fue considerada como especie dominante, así como el zacate Johnson $(S$. halepense) lo fue en el ciclo 1991-92 (Cuadro 2).

En el ciclo 1990-91, los mejores controles de $M$. divaricatum se obtuvieron con las dosis mas altas de alaclor y linurón y las tres dosis de fomesafén. Ninguno de los herbicidas controló a $C$. rotundus; solamente se notaron ligeros efectos temporales con la dosis alta de

Cuadro 2. Densidad de población de malezas en los sitios experimentales. CIRGOC. INIFAP. SAGAR.

\begin{tabular}{|c|c|c|c|c|c|}
\hline \multirow[t]{3}{*}{ Nombre científico } & \multirow[t]{3}{*}{ Familia } & \multicolumn{4}{|c|}{ Población/ha (miles) } \\
\hline & & \multicolumn{2}{|c|}{ Experimento 1990-91 } & \multicolumn{2}{|c|}{ Experimento 1991-92 } \\
\hline & & 15 DDE & 30 DDE & 15 DDE & 30 DDE \\
\hline Melampodium divaricatum (Rich.) DC. & Compositae & 1.510 & 1.370 & 360 & 240 \\
\hline Cyperus rotundus $\mathrm{L}$. & Cyperaceae & 480 & 620 & 260 & 570 \\
\hline Euphorbia heterophylla $\mathrm{L}$. & Euphorbiaceae & - & - & 350 & 550 \\
\hline Sorghum halepense (L.) Pers. & Gramineae & 190 & 280 & - & - \\
\hline Cynodon dactylon (L.) Pers. & Gramineae & - & - & 230 & 250 \\
\hline Lagascea mollis (Cav.) & Compositae & - & 260 & - & - \\
\hline Parthenium hysterophorus L. & Compositae & - & - & 160 & 220 \\
\hline Echinochloa colona (L.) Link & Gramineae & 170 & 20 & - & - \\
\hline Tragia mexicana Muell. & Euphorbiaceae & - & - & 150 & 70 \\
\hline Argemone mexicana $\mathrm{L}$. & Papaveraceae & 50 & - & 70 & 40 \\
\hline Kallstroemia maxima (L.) T. \& C. & Zygophyllaceae & - & 10 & 40 & 40 \\
\hline Euphorbia hirta L. & Euphorbiaceae & 90 & - & - & - \\
\hline Panicum fasciculatum Sw. & Gramineae & - & - & - & 20 \\
\hline Commelina diffusa Burm. $\mathrm{f}$. & Commelinaceae & - & 10 & - & - \\
\hline
\end{tabular}


alaclor, las tres dosis de fomesafén, y la mezcla fluazifopbutil + fomesafén. A su vez, S. halepense solo fue controlado con la mezcla antes indicada, ya que fluazifopbutil es un herbicida específico para el control de gramíneas (Cuadro 3).

Ninguno de los tratamientos preemergentes causó toxicidad al frijol. Sin embargo, todos los tratamientos aplicados en postemergencia ocasionaron toxicidad, cuya severidad varió entre el 21 y $34 \%$ en la primera época de evaluación y 13 y $22 \%$ en la segunda época (Cuadro 3)).

El testigo limpio tuvo un rendimiento de grano significativamente superior al resto de los tratamientos. Entre los tratamientos químicos, linurón ( $2 \mathrm{~kg} / \mathrm{ha}$ ) tuvo el rendimiento más alto, aunque éste fue estadísticamente semejante a otros ocho tratamientos, incluyendo el testigo enhierbado (Cuadro 3). Cabe señalar que en este ciclo, la humedad residual disponible en el terreno fue muy escasa, lo que ocasionó que los rendimientos obtenidos, incluyendo el testigo limpio fueran más bajos que los que normalmente se obtienen en la región.

En el ciclo 1991-92, alaclor tuvo controles del 65\% o menores, tanto de $M$. divaricatum, como de $E$. heterophylla. Linurón en sus dos dosis, controló mas eficientemente las especies antes indicadas, si bien la dosis más alta, solamente produjo un $81 \%$ de control en la primera época de evaluación. Las mezclas de alaclor + linurón tuvieron un control inicial ligeramente mayor que el obtenido con linurón, pero en la segunda evaluación sus controles fueron semejantes o inferiores a éste (Cuadro 4).

Clortal-dimetil solamente mostró un ligero efecto sobre las malezas de hoja ancha en la primera evaluación, pero éste desapareció en la segunda evaluación. Por su parte, las dos dosis de fomesafén fueron las más eficientes en el control de estas dos especies, teniendo controles entre 92 y $97 \%$ en la primera evaluación y 87 y $95 \%$ en la segunda (Cuadro 4).

A excepción de fomesafén, que mostró un ligero efecto sobre $C$. rotundus, el resto de los tratamientos no lo afectó en lo absoluto (Cuadro 4).

Tanto alaclor como linurón ocasionaron toxicidad al frijol, siendo relativamente mayor con la dosis alta de linurón, al grado de ser el tratamiento con el segundo rendimiento mas bajo. En la primera evaluación, la toxicidad ocasionada por fomesafén, fue significativamente menor que la reportada en el ciclo 1990-91, y para la segunda evaluación, las plantas prácticamente no mostraron efectos tóxicos (Cuadro 4).

El rendimiento de grano más alto, se obtuvo en el testigo limpio, siendo estadísticamente semejante al

Cuadro 3. Efecto de aplicaciones de herbicidas en el control de maleza, toxicidad y rendimiento del frijol de humedad residual en el ciclo O-I 1990-91. CIRGOC. INIFAP. SAGAR.

\begin{tabular}{|c|c|c|c|c|c|c|c|c|}
\hline \multirow[t]{2}{*}{ Trat. } & \multirow{2}{*}{$\begin{array}{c}\text { Dosis } \\
\text { (kg i.a./ha) }\end{array}$} & \multirow{2}{*}{$\begin{array}{l}\text { Epoca de } \\
\text { aplic. }\end{array}$} & \multicolumn{3}{|c|}{ Porcentaje de control } & \multirow{2}{*}{$\begin{array}{c}\text { Toxic. } \\
\text { frijol } \\
\text { 1e-2e }\end{array}$} & \multirow{2}{*}{$\begin{array}{l}\text { Rend. } \\
\text { (kg/ha) }\end{array}$} & \multirow{2}{*}{$\begin{array}{c}\text { Duncan } \\
(0,05)\end{array}$} \\
\hline & & & $\begin{array}{l}\text { M.d. } \\
\text { e-2e }\end{array}$ & $\begin{array}{l}\text { C.r. } \\
\text { 1e-2e }\end{array}$ & $\begin{array}{l}\text { S.h. } \\
\text { 1e-2e }\end{array}$ & & & \\
\hline Alaclor & 0,44 & PRE & $28-15$ & $0-0$ & $0-0$ & $0-0$ & 233,1 & bcd \\
\hline Alaclor & 1,5 & PRE & $68-45$ & $0-0$ & $0-0$ & $0-0$ & 191,1 & $\mathrm{~cd}$ \\
\hline Alaclor & 2 & PRE & $90-80$ & $18-0$ & $0-0$ & $0-0$ & 244,2 & bcd \\
\hline Linurón & 1 & PRE & $50-45$ & $0-0$ & $0-0$ & $0-0$ & 212,8 & bcd \\
\hline Linurón & 1,5 & PRE & $82-68$ & $0-0$ & $0-0$ & $0-0$ & 253,9 & bcd \\
\hline Linurón & 2 & PRE & $94-92$ & $0-0$ & $0-0$ & $0-0$ & 345,7 & $\mathrm{~b}$ \\
\hline $\mathrm{F}-\mathrm{B}+\mathrm{F}$ & $0,25+0,25$ & $15 \mathrm{DDE}$ & $100-98$ & $13-3$ & $98-84$ & $34-14$ & 167,2 & $\mathrm{~d}$ \\
\hline Fomesafén & 0,187 & $15 \mathrm{DDE}$ & $100-97$ & $10-1$ & $0-0$ & $21-13$ & 299,2 & $\mathrm{bc}$ \\
\hline Fomesafén & 0,25 & $15 \mathrm{DDE}$ & $100-98$ & $13-0$ & $0-0$ & $31-18$ & 229,7 & bcd \\
\hline Fomesafén & 0,375 & $15 \mathrm{DDE}$ & $100-99$ & $13-1$ & $0-0$ & $34-22$ & 184,7 & $\mathrm{~cd}$ \\
\hline T. L. & $100-100$ & $100-100$ & $100-100$ & $0-0$ & 517,4 & $\mathrm{a}$ & & \\
\hline T. E. & $0-0$ & $0-0$ & $0-0$ & $0-0$ & 232,8 & bcd & & \\
\hline
\end{tabular}

M.d. = Melampodium divaricatum; C.r. = Cyperus rotundus; S.h. = Sorghum halepense;

$1 \mathrm{e}=$ primer evaluación; $2 \mathrm{e}=$ segunda evaluación; $\mathrm{PRE}=$ preemergencia; $\mathrm{DDE}=$ días después de la emergencia; $\mathrm{F}-\mathrm{B}+\mathrm{F}=$ Fluazifop-Butil + Fomesafén; TL = testigo limpio; TE = testigo enhierbado 
Cuadro 4. Efecto de aplicaciones de herbicidas en el control de maleza, toxicidad y rendimiento del frijol de humedad residual en el ciclo O-I 1991-92. CIRGOC. INIFAP. SAGAR.

\begin{tabular}{|c|c|c|c|c|c|c|c|c|}
\hline \multirow[t]{2}{*}{ Trat. } & \multirow{2}{*}{$\begin{array}{c}\text { Dosis } \\
\text { (kg i.a./ha) }\end{array}$} & \multirow{2}{*}{$\begin{array}{c}\text { Epoca de } \\
\text { aplic. }\end{array}$} & \multicolumn{3}{|c|}{ Porcentaje de control } & \multirow{2}{*}{$\begin{array}{l}\text { Toxic. } \\
\text { frijol } \\
1 e-2 e\end{array}$} & \multirow{2}{*}{$\begin{array}{l}\text { Rend. } \\
\text { (kg/ha) }\end{array}$} & \multirow{2}{*}{$\begin{array}{c}\text { Duncan } \\
(0,05)\end{array}$} \\
\hline & & & $\begin{array}{l}\text { M.d. } \\
\text { 1e-2e }\end{array}$ & $\begin{array}{c}\text { E.h. } \\
\text { 1e-2e }\end{array}$ & $\begin{array}{c}\text { C.r. } \\
\text { 1e-2e }\end{array}$ & & & \\
\hline Alaclor & 2 & PRE & $65-40$ & $53-35$ & $0-0$ & $8-5$ & 661 & def \\
\hline Alaclor & 2,5 & PRE & $60-54$ & $58-48$ & $0-0$ & $8-3$ & 892 & cde \\
\hline Clortal DM & 6 & PRE & $0-0$ & $0-0$ & $0-0$ & $0-0$ & 656 & def \\
\hline Clortal DM & 7,5 & PRE & $15-0$ & $15-0$ & $0-0$ & $0-0$ & 453 & $\mathrm{f}$ \\
\hline Linurón & 1,5 & PRE & $74-71$ & $71-69$ & $0-0$ & $16-11$ & 969 & $\mathrm{~cd}$ \\
\hline Linurón & 2 & PRE & $81-74$ & $80-68$ & $0-0$ & $48-35$ & 542 & ef \\
\hline $\mathrm{Ala}+\mathrm{Lin}$ & $1+0,5$ & $15 \mathrm{DDE}$ & $86-65$ & $81-64$ & $0-0$ & $0-0$ & 884 & cde \\
\hline $\mathrm{Ala}+\mathrm{Lin}$ & $1,5+0,5$ & $15 \mathrm{DDE}$ & $88-64$ & $88-69$ & $0-0$ & $0-0$ & 831 & de \\
\hline Fomesafén & 0,25 & $15 \mathrm{DDE}$ & $93-90$ & $92-87$ & $14-0$ & $16-0$ & 1191 & $\mathrm{bc}$ \\
\hline Fomesafén & 0,375 & $15 \mathrm{DDE}$ & $95-95$ & $97-95$ & $23-0$ & $19-3$ & 1351 & $\mathrm{ab}$ \\
\hline T. L. & $100-100$ & $100-100$ & $100-100$ & $0-0$ & 1666.5 & $\mathrm{a}$ & & \\
\hline T. E. & $0-0$ & $0-0$ & $0-0$ & $0-0$ & 668.0 & def & & \\
\hline
\end{tabular}

*/ M.d. = Melampodium divaricatum; E.h. = Euphorbia heterophylla $;$ C.r. = Cyperus rotundus;

$1 \mathrm{e}=$ primer evaluación; $2 \mathrm{e}=$ segunda evaluación; $\mathrm{PRE}=$ preemergencia; $\mathrm{DDE}=$ días después de la emergencia; $\mathrm{Clortal} \mathrm{DM}=$ clortal dimetil; Ala + Lin = alaclor + linurón; $\mathrm{TL}=$ testigo limpio; $\mathrm{TE}=$ testigo enhierbado

tratamiento de fomesafén a $0,375 \mathrm{~kg} / \mathrm{ha}$, que a su vez lo fue a fomesafén a $0,25 \mathrm{~kg} / \mathrm{ha}$. En el resto de los tratamientos, el rendimiento fue comparable al del testigo enhierbado, que produjo $668 \mathrm{~kg}$ de frijol/ha (Cuadro 4).

$\mathrm{Al}$ analizar la información de los dos experimentos, se observó que la eficiencia y en algunos casos la toxicidad de los herbicidas fue afectada por las condiciones de humedad residual en el terreno. Por ejemplo, tanto alaclor como linurón tuvieron un mejor control de $M$. divaricatum cuando la humedad residual fue mas baja. Asimismo, la toxicidad de linurón al frijol, se presentó solamente cuando se tuvieron buenas condiciones de humedad en el terreno.

En el caso del fomesafén, los controles de $M$. divaricatum no tuvieron una variación tan marcada cuando este herbicida se aplicó en condiciones de baja o buena humedad del terreno. Por lo anterior, puede considerarse que el fomesafén representó una buena opción para el control de malezas de hoja ancha en frijol, que son junto con $C$. rotundus las especies más importantes de este cultivo en la región central del estado de Veracruz (Acosta, 1979).

Aunque $C$. rotundus no fue controlado por ninguno de los tratamientos evaluados, en un experimento efectuado con anterioridad, se determinó que esta maleza puede controlarse con el herbicida bentazón. Este último herbicida también puede utilizarse para el control de
M. divaricatum, pero no de E. heterophylla, ni de algunas especies de Amaranthus (Esqueda, Acosta y Durán, 1981). Así pues, el uso del bentazón o el fomesafén debe decidirse de acuerdo a la composición de la población de malezas presentes en el terreno.

Por lo general, las gramíneas no se presentan como un problema de consideración para el frijol, pero de ser así (como S. halepense en el ciclo 1990-91), éstas pueden controlarse con $0,25 \mathrm{~kg} / \mathrm{ha}$ de fluazifop-butil.

\section{CONCLUSIONES}

1. En preemergencia, los mejores controles de $M$. divaricatum se obtuvieron con alaclor $(2 \mathrm{~kg} / \mathrm{ha})$ y linurón $(2 \mathrm{~kg} / \mathrm{ha})$, sin embargo, la eficiencia y toxicidad de estos productos varió grandemente de acuerdo al nivel de humedad residual del terreno.

2. En postemergencia, fomesafén $(0,25 \mathrm{~kg} / \mathrm{ha})$ tuvo un control eficiente tanto de M. divaricatum como de E. heterophylla.

3. Alaclor mostró un control deficiente de $E$. heterophylla, incluso con la dosis de $2,5 \mathrm{~kg} / \mathrm{ha}$.

4. Ninguno de los tratamientos evaluados controló a C. rotundus. 


\section{LITERATURA CITADA}

ACOSTA, S. 1979. Reconocimiento de la maleza en el cultivo de frijol (Phaseolus vulgaris) en la región de la Mixtequilla, Veracruz. Centro de Investigaciones Agrícolas del Golfo Centro. SARH. INIA. 12 p.

AGUNDIS , O.; VALTIERRA, A.; CASTILLO, B. 1962. Períodos críticos de competencia entre frijol y malezas. Agricultura Técnica en México 2(2):87-90.

BARRETO, A. 1970. Competencia entre frijol y malas hierbas. Agricultura Técnica en México 2(12):519-526.

CANO, O.; LÓPEZ, E. 1996. Control preemergente y postemergente de malezas en frijol, de humedad residual en Veracruz, México. Agronomía Mesoamericana $7(2): 4249$.

ESQUEDA, V.A; DURAN, A. 1992. Efecto de la época y tipo de limpia en el rendimiento del frijol de humedad residual. In: Memorias XIII Congreso Nacional de la Ciencia de la Maleza. Chapingo, edo. de México, 11-13 de noviembre de 1992.p.56.

ESQUEDA, V. A.; ACOSTA , S.; DURÁN , A. 1981. Evaluación de herbicidas en frijol de humedad residual en la región central del estado de Veracruz. In: Memorias II Congreso Nacional de la Ciencia de la Maleza. Chapingo, edo. de México, 4 al 7 de noviembre de 1981.p. 200-212.
GARCÍA, M. J. T.; BARRALES, D. S. 1988. Evaluación de herbicidas postemergentes en el cultivo del frijol(Phaseolus vulgaris L.) bajo condiciones de temporal en Santa María Zacatepec, Puebla. Revista Chapingo 59:50-53.

OBANDO, A. J. 1981. Las malezas y su control en el cultivo del frijol en la región de General Trías- Satevo, Chih. p. 179-199. In: Memorias del II Congreso Nacional de la Ciencia de la Maleza. Chapingo, edo. de México, 4 al 7 de noviembre de 1981.

ROMERO, D.; ALEMÁN, F. 1990. Determinación de dosis y momento óptimo de aplicación de los herbicidas Fomesafén y Fluazifop-butil en el control postemergente de malezas en frijol común (Phaseolus vulgaris L.). Revista de la Escuela de Sanidad Vegetal 1:22-30.

THOMSON, W. T. 1993. Agricultural Chemicals Book II herbicides. Fresno, Thomson Publications. 310 p.

WILSON, R. G. JR.; WICKS, G. A.; FENSTER, C. R. 1980. Weed control in field beans (Phaseolus vulgaris) in Western Nebraska. Weed Sci. 28:295-299. 